\title{
Quantum-Enhanced Microwave Backscattering Communications
}

\author{
Riku Jäntti, Ruifeng Duan, Jari Lietzén, Hany Khalifa, and Lajos Hanzo
}

\begin{abstract}
The recent advances in the field of microwave superconducting circuits open the way for a multitude of engineering applications that revolutionize the field of classical communications. To exploit this new technology, we propose a novel microwave quantum-enhanced backscattering system based on the laws of quantum physics. Both the transmitter and the receiver are quantum mechanical in nature and are accommodated at the infrastructure side, while the backscattering device is classical. The advocated system breaks the performance barrier of the classical backscattering systems and approaches the ultimate attainable receiver sensitivity. Finally, our quantum solution outperforms the classical solutions in terms of its level of security.
\end{abstract}

\section{INTRODUCTION}

Quantum solutions rely on the amalgam of physics and engineering, exploiting quantum entanglement, quantum superposition and quantum tunnelling for compelling practical applications, such as quantum communications. Recently, quantum key distribution [1], [2], and quantum error correcting codes [3] have attracted substantial research interest. In addition, quantum location verification (QLV) has been suggested for enhancing the security of the driverless vehicles of the near future [4]. Furthermore, quantum-secure imaging [5] holds the promise of realizing secure engineering systems that are robust against jamming.

However, relatively little attention has been dedicated to wireless applications. Quantum-assisted wireless communication research seeks to invoke quantum computers for enhancing the digital baseband processing at the receiver. The components of this new system are manufactured from superconducting elements, which lend themselves to convenient practical implementation. Consequently, attractive applications of quantum-engineering techniques to wireless communications have started to emerge [6].

Riku Jäntti, Ruifeng Duan, Jari Lietzén and Hany Khalifa are with Aalto University. Lajos Hanzo is with the University of Southampton.

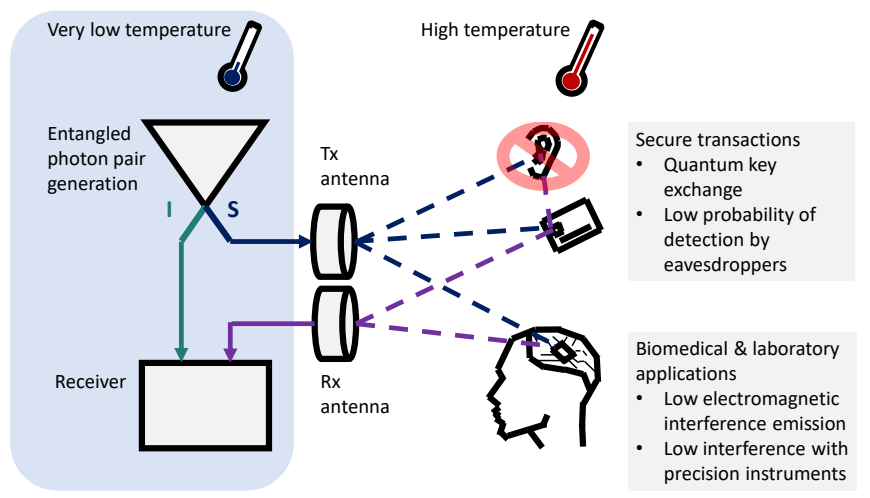

Fig. 1. Proposed system for QBC together with potential applications.

Most of the applications of quantum technology rely on quantum entanglement as a resource, which implies that the spin of an entangled pair of electrons changes instantaneously in an intrinsically coupled manner, regardless of their physical distance 1 However, entanglement is a fragile state of the associated particles, which may be destroyed by the deleterious effects of decoherence even in the absence of other impairments introduced by the wireless channel. Despite this fact, the surviving entangled pairs can prove useful in support of the quantum illumination (QI) concept of Lloyd conceived in 2008 [7], which increases the detection probability of a low-reflectivity object even in a hostile lossy environment. Explicitly, QI exploits the residual correlation between the two initially entangled systems even if the entanglement itself may not survive. Its exciting applications in the microwave regime [8] have paved the way for a quantum radar prototype [9].

Backscattering refers to reflection of signals back to the direction which they came from. Radars use backscattering to detect objects, while Radio

\footnotetext{
${ }^{1}$ This phenomenon was referred to as a 'spooky action at a distance' by Einstein, which however should not be interpreted as a violation of the speed of light, because a number of preparatory operations have to be carried out first.
} 
Frequency (RF) Identification (RFID) systems use it to read the identity of a passive RF-tag. Quantum backscatter communications (QBC) [10], [11] can be interpreted as a compelling application of quantum radar to communications in a way analogous to RFID applying the classical radar principle for transmitting the tag's identity. In fact, the system architecture of QBC is simpler than that of the quantum radar, since a backscatter antenna is a spatially fixed object. Thus it can be more readily manipulated than a quantum radar target. As a further benefit of its improved tolerance against noise and owing to the ultra-low radiated power, QBC can share its frequency band with a legacy system without imposing excessive interference.

Again, microwave quantum technology relies on superconducting elements, hence both the transmitter and the receiver of our QBC system have to operate within a cryogenic chamber. Fortunately in the backscattering scenario considered, all quantum components can be accommodated by a Reader a.k.a. Base Station (BS) as seen in Fig.1. Thus, all the cryogenic components are part of a sophisticated Reader/BS infrastructure, making the deployment of the system feasible.

The low-radiated power used by QBC minimizes the electromagnetic interference emissions, which is particularly attractive in medical applications. Lowpower operation is also of paramount importance in industrial environments having a risk of explosion. Moreover, the enhanced security of QBC is beneficial in applications, where the confidentiality of the information is a salient criterion. Such applications can be found in health-care, banking, industrial and governmental sectors. We note here that our discussions are not limited to any particular frequency. Practical means of generating microwave photons are available at least for the $5-20 \mathrm{GHz}$ range. The QBC systems and their potential applications are illustrated in Fig. 1. In this article, we will first introduce the QBC paradigm and discuss its performance, implementational options and open challenges. Finally, we offer tangible conclusions, discuss a range of open research questions and promising future approaches.

\section{QUANTUM BACKSCATTER COMMUNICATION}

Again, QBC achieves its enhanced sensitivity by exploiting the quantum domain correlations to capture faint backscattered microwave signals. The typical setup of QBC involves a transmitter that generates entangled photon pairs, where one of the photons is utilized for illuminating the backscattering antenna, while the other is retained at the receiver in a memory element that can be accurately modeled by a pure lossy channel, which is co-located with the Reader/BS transmitter of Fig. 1. The backscattering antenna is assumed to be immersed in a noisy and lossy environment. In the next section we describe the operational details of each component of our QBC system of Fig. 1.

\section{A. QBC system components}

The QBC system of Fig.1 consists of two parts: a reader/BS and a tag. The reader/BS is placed in a quantum circuit refrigerator that keeps the operating temperature below $20 \mathrm{mK}$ (milli-Kelvin). Superconducting microwave circuits are utilized to generate entangled signal (S) - idler (I) photon pairs. The propagating microwave signal photon is then forwarded to the antenna, which is outside the refrigerator. The tag is a classical device, namely a simple antenna having a controllable reflection coefficient, placed in a thermal bath having temperatures ranging from $3 \mathrm{~K}$ (space) to $300 \mathrm{~K}$ (room temperature). The transmitted signal photon interacts with the tag antenna and it is then scattered back towards the Reader/BS receiver antenna.

Assuming a linear lossy medium, the interaction between the transmitted microwave (or optical) photons passing through the channel can be modeled by a simple beam-splitter that mixes the transmitted signal photons with thermal noise. A beam-splitter is an optical device that has two inputs and outputs. Each input beam gets split among the two output ports thus mixing the signals. Beam-splitters operating at microwave frequencies are also available. At the receiver the quantum advantage manifests itself through the detection method adopted. A lowcomplexity detector relies on performing a joint measurement to both the signal and the idler modes using a balanced beamsplitter [12]. When the pair of indistinguishable signal and idler photons impinge on a balanced beamsplitter from two different ports, they coalesce and exit the beamsplitter from the same port. Experimentally, this can be verified by counting the associated coincidence events. The pair of physical processes that lead to a coincidence count are either of a reflection-reflection or of a transmission-transmission nature. On a balanced 


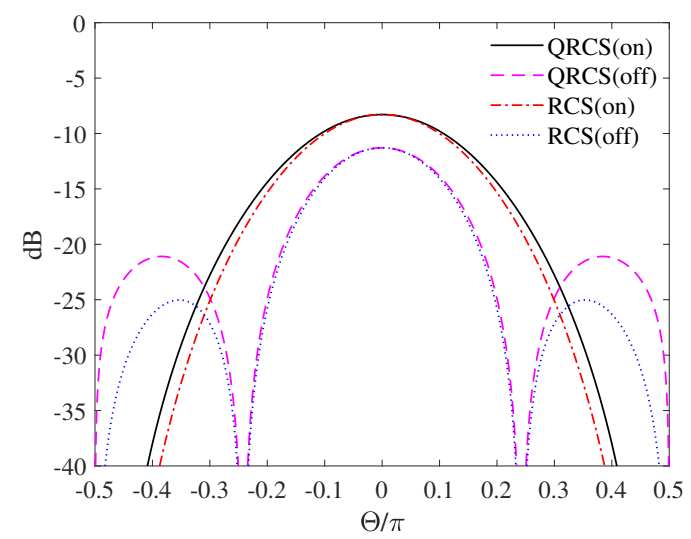

Fig. 2. Quantum radar cross-section and radar cross-section of a backscatter antenna viewed from angle $(\Theta, 0)$.

beamsplitter these two processes destructively interfere as they become indistinguishable, thus leading to a zero coincidence rate. Classically, the coincidence probability is unity since classical signals are unable to interfere quantum-mechanically as single photons. Hence, a quantum detection scheme can distill the transmitted signal from the deleterious effects of environmental noise in a manner superior to the classical detection methods.

In the quantum radar literature [9], round trip transmissivity (RTT) $\eta$ of the channel models all the losses encountered. In free-space, the RTT, $\eta$, imposed by the channel having the target present is directly proportional to the radar cross section (RCS) and inversely proportional to the fourth power of the distance from the Reader/BS to the target. The RTT also depends on other parameters such as the transceiver antenna gain and the frequency utilized [9]. In classical radar, the impact of the target is characterized by the classical RCS (CRCS) parameter. In backscatter communications, the CRCS depends both on the antenna construction and on the geometry of the backscatter communication device. In the case of quantum radar, the corresponding parameter is termed as the quantum RCS (QRCS). A method of calculating the QRCS for a simple metallic object can be found in [9].

Fig. 2 shows the QRCS and CRCS gain versus the angle $\Theta$ in the $X-Z$ plane for a simple dipole metal plate antenna construction shown in Fig. 3. The antenna modulates and transmits the signal photons by using a mechanical switch. When the switch is open, there is an air-gap between the two metal plates. When the switch closes, the

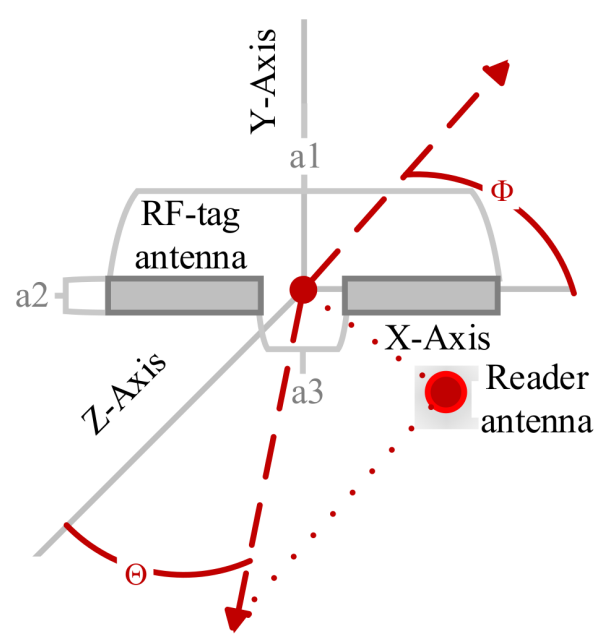

Fig. 3. An illustration of the geometry of the Reader/BS-Tag system. The backscatter antenna is viewed from an angle $(\Theta, \Phi)$.

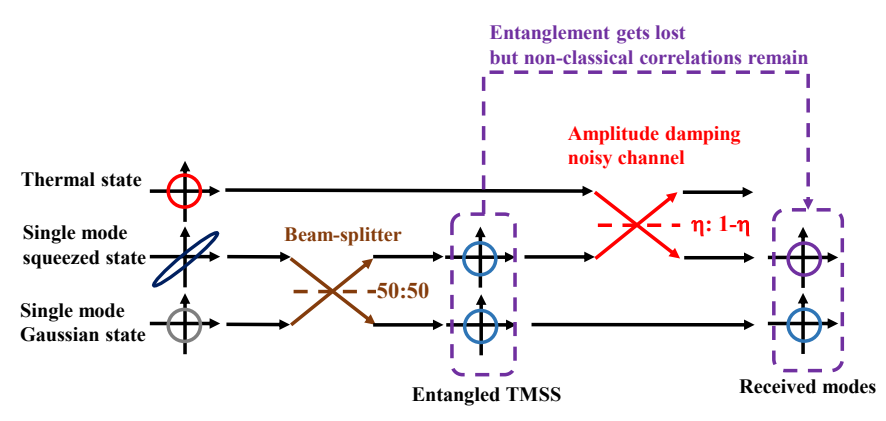

Fig. 4. A visual illustration of a coherent state, a squeezed state, TMSS, quantum correlations, and their relationship.

antenna functions as a single rectangular metal plate. By controlling the switch, simple binary pulse amplitude modulation (PAM), also known as onoff-keying (OOK), can be performed by switching between a pair of uniquely distinguishable values of RTT (defined by two different QRCS values). In Fig. 3, the parameters $a_{1}, a_{2}, a_{3}$ define the antenna dimensions.

For illustrative purposes, we choose the antenna shown in Fig. 2 to characterize the difference between the QRCS and the CRCS. In Fig. 2 the antenna dimensions were chosen to obtain a $3 \mathrm{~dB}$ difference between the on and off states. It can be seen from Fig. 2 that if the antenna is viewed directly from the front, there is no difference between the QRCS and the CRCS. By contrast, when the antenna is viewed at an angle, the QRCS is higher than the CRCS. This implies that the antenna becomes more visible in the quantum setting. Unfortunately, this also indicates that any clutter in the environment 
becomes more hostile, which in turn degrades the performance of the system. Although the channel destroys the strictly coherent entanglement between the I and the received S photons, some residual nonclassical correlations still remain. Again, QI [7] is a protocol that improves the detector's (classifier's) performance in the face of channel impairments by exploiting these correlations.

\section{B. Quantum illumination protocol}

For the sake of simplifying our problem, we consider an experimental setup, where the average number of noise photons $N_{z}$ per each coupled environmental mode is small. Quantitatively, the average number $N_{z}$ of noise photons per measurement is less than one, regardless of its frequency or polarization. We also assume that the RTT is small, so that the number of signal photons per measurement is even lower. Hence the overall 'signal-to-noise ratio' (SNR) is very low. Consequently, the number of photons (probe intervals) $M$ required for reliably distinguishing between the state 'on' and 'off' is relatively large.

In $\mathrm{QI}$, the detection is carried out by applying a joint measurement to the backscattered signal photon $\mathrm{S}$ and the idler photon I for exploiting the quantum correlation between them to distill the desired signal, separating it from the noise. In comparison to sending unentangled single photons, this protocol improves the SNR of the detector by a factor of $d=2^{m}$, where $m$ denotes the number of entangled photon pairs [7].

The impact of QI is equivalent to reducing the noise by the factor of $d$, hence the number of photons required for reliable detection is also reduced by a factor of $d$. Practically, when the idler photon is totally lost, any quantum advantage disappears.

In classical backscattering communications, a Reader/BS uses a voltage controlled oscillator to generate an unmodulated sinusoidal carrier. The quantum mechanical analog for it is the quantum harmonic oscillator. Measurements of the in-phase and quadrature components of the generated signal follow the Gaussian distribution. Measuring the field quadratures reveals that their probability distribution follows a Gaussian distribution. The states characterized by a Gaussian probability distribution are also often termed as minimum uncertainty states. Coherent states constitute examples of minimum uncertainty states, where the variance in the quadrature measurement is minimum. Another interesting minimum uncertainty state is the so-called squeezed state. To elaborate, in contrast to a coherent state, the uncertainties in the field quadratures of a squeezed state are not equal, but nonetheless their product is the same as that of a coherent state. Operationally this means that the uncertainty in one of the quadratures is reduced at the expense of the other in order to keep their product value as that of a minimum uncertainty state. The Two Mode Squeezed State (TMSS) represents a continuousvariable entanglement between two modes of a radiation field that can be utilized in both the optical and microwave domains. Fig. 4 depicts a visual illustration of a coherent state, a squeezed state, TMSS, quantum correlations, and their relationship.

\section{Performance}

In quantum radar, the logical states are the 'target absent' and 'target present', which correspond to binary PAM, also known as OOK in backscatter communications. This scheme can be realized at the Tag using a single switch adjusting a load impedance. Nonetheless more complex modulation schemes such as binary phase shift keying (BPSK), quadrature phase shift keying (QPSK), multi-level PAM and quadrature amplitude modulation (QAM) can also be constructed by appropriately designing the backscatter communications modulator circuitry. In a number-state scenario, the receiver is based on photon counting and has no direct access to the channel phase. By contrast, in the Gaussian-state scenario, the quadradure carriers are measured and the phase becomes readily available. The down-side of the latter approach is that the channel-induced phase changes have to be estimated. Typically a large number of mode pairs $M$ is required for reliably transmitting a bit over the channel. Ideal quantum measurement chosen for demodulation will use all these $M$ mode pairs simultaneously, which relies on quantum memory. Since having a large quantum memory storing millions of mode pairs is impractical, we have to resort to sub-optimal receiver designs. Because the theoretical calculation of the bit error probability for the ideal receivers is not tractable, we adopt the Quantum Chernoff Bound (QCB) for the bit error probability, which decays exponentially with $\zeta \cdot M$, where $\zeta$ is a pa- 
rameter proportional to the $\mathrm{SNR}$. The QCB becomes asymptotically tight as $M$ approaches infinity.

Figure 5 shows some preliminary results from [11] based on the quantum Chernoff bound for the achievable bit error rate of different modulation methods and receiver structures. We compare a coherent receiver $(\mathrm{C})$ that does not apply QI using a Parametric Amplifier (PA) based receiver and a Sequential Frequency Generation (SFG) receiver [13]. The analysis is performed based on TMSS. The system was able to generate entangled mode pairs at rate $N_{s}$ and encountered on average $N_{z}$ noise photons per mode. The ideal SFG receiver achieves the full $6 \mathrm{~dB}$ gain over the coherent receiver for PAM and BPSK, but only $3 \mathrm{~dB}$ for QPSK. The PA receiver achieves $3 \mathrm{~dB}$ gain over coherent PAM and BPSK, but provides no gain for QPSK. Having the phase information available provides $6 \mathrm{~dB}$ gain for BPSK over PAM, just like in classical backscatter communications. Indeed, to the best of our knowledge the $6 \mathrm{~dB}$ gain is a theoretical limit, with no known realization at the time of writing.

\section{Security}

A secure protocol using QI has been proposed in [1] for optical communications. The scheme can be adapted to QBC as well. As discussed above, using QI may provide 3 - $6 \mathrm{~dB}$ gain in the error exponent for the communication link (a.k.a. Bob-Alice) between the backscatter device Bob and the source transmitter Alice compared to the link between Bob and the eavesdropping receiver (referred to as BobEve). This is due to the fact that Eve has no access to the idler photons kept at the receiver. In order for Eve to compensate for this, she would have to use higher-gain antennas than Alice or be closer to Bob than Alice. If Alice uses TMSS to generate the Signal-Idler mode pairs and Bob applies BPSK modulation, then Alice can further increase the link security by $2 \mathrm{~dB}$ upon applying random phase shifts to both the signal and idler paths, hence making it impossible for Eve to use non-coherent reception.

QBC is vulnerable to active eavesdropping attacks, where Eve will illuminate Bob's antenna with its own signal photons. Hence, Eve's signal will cause interference at Alice's receiver, which can be detected by observing the long-term photoncounting statistics. Adding a photon detector at Bob's receiver can also be utilized to detect Eve's malicious actions, but in practice this implies that a fraction of the power impinging on Bob's antenna has to be fed to the detector, thus reducing the QRCS (and hence $\eta$ ). For instance, 50-50 power split at Bob's antenna reduces the error exponent by $3 \mathrm{~dB}$, but this equally affects both Alice and Eve. We note, however, that in the microwave domain the transmission range, over which acceptable secure key generation rates can be achieved, is on the order of centimeters [2]. This reduces the applicability of the protocol to near field communications (NFC) based tag reading.

\section{Challenges And SOlution APPROACHES}

\section{A. Number of photons generated}

In contrast to an avionic quantum radar that is pointed towards the sky, the naval quantumradar systems and the QBC systems are typically pointed towards the earth and thus operate in a much brighter thermal background. To compensate for the loss $\eta$ caused by the antennas not capturing all the transmitted photons and the high number of thermal photons $N_{z}$, a large number of entangled mode pairs $M$ has to be generated. The parameter $M$ is directly proportional to the bandwidth $W$ and the utilized pulse length $T_{s}$. The current microwave photon sources can generate approximately $10^{6}$ photons per second. However, we need approximately $10^{9}$ photons for reliable detection. In [14], the socalled 'virtual' modes were introduced by using several QI devices for illuminating the same target in parallel. This approach suffers from mutual interference between the Readers/BSs, since the signal photons generated by neighboring Readers are not correlated with the idler photons used by the Reader to enhance the detection/demodulation. The interference can be mitigated by using beamsplitters to perform analog beam-forming [10]. In the idea, $N$ reader and $N$ antenna system, the beamforming system can obtain a factor $N^{2}$ gain over a single reader system. The number of mode pairs we need for reliable demodulation is a factor 1000 smaller. We can compensate for this by using a 32antenna system.

\section{B. Impact of clutter}

Naturally, reflection and clutter caused by objects other than the Tag antenna will also be present at the receiver. The photon propagation in cluttered environment can be modeled by a random walk 


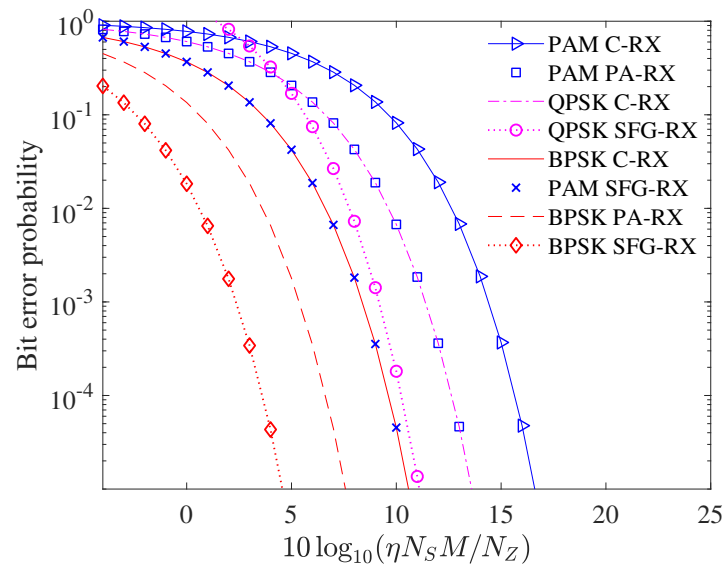

Fig. 5. Bit error rate as a function of $\zeta=\eta N_{s} M / N_{z}$.

from one object to another. We consider a simple model consisting of a single-bounce paths, where a photon either directly reaches the Tag antenna or hits a cluttering object in between. If the number of objects is large, then we can model the channel $\sqrt{\eta}$ as a complex Gaussian random variable. In the absence of a direct path (zero mean), the model becomes equivalent to Rayleigh fading. However, if the channel has a strong line-of-sight component, the channel exhibits Rician fading, which is characterized by two parameters, the Rice factor and the total received intensity (power). The main difference between the quantum fading channel and the classical one arises from the QRCS. Assuming that the main lobe of the Tag antenna is directed towards the Reader/BS antenna, the ratio of the classical and quantum Rice factors is $\pi / 4$, which is less than 1. This indicates that the QBC system experiences more severe fading than its classical counterpart. Multi-antenna techniques and forward error correction coding is required to mitigate its effect.

\section{Channel estimation}

Coherent demodulation techniques require the knowledge of the phase shift caused by the channel. In backscattering communications, we can send known pilot symbols for estimating the channel. In a quantum protocol, the phase uncertainty is inversely proportional to the number of photons used, namely $M$, which is a factor of square-root $M$ better than in classical cases. Due to the high loss in the channel, the number of photons needed will nevertheless be large. Given the challenge of generating enough photons, protocols avoiding the need for channel estimation are preferable.

\section{Coding and Achievable Rate}

Since the considered QBC system is likely to operate at low SNR, ideally we should use forward error correction (FEC) coding techniques for improving the performance. Lower bounds for the capacity of QI-assisted optical communications in case of BPSK modulation and PA receiver have been derived in [1], when $M$ mode pairs were utilized per symbol. The results can be adapted to microwave scenarios as well. Assuming that the bit error rate would follow QCB, we could achieve a capacity of $C \approx 0.76$ bits/channel-use employing $M \approx 2.81 / \zeta$ photons per channel-use.

\section{E. Practical implementation considerations}

Quantum Illumination has been recently demonstrated experimentally at the microwave frequencies [15]. The experimental system generated entangled fields using a Josephson parametric converter at milli-Kelvin temperatures to illuminate a roomtemperature object at a distance of 1 meter in a proof of principle bistatic radar setup. The experimental setup utilized heterodyne measurement in $300 \mathrm{~K}$ temperature to study the achievable SNR gain of QI. Owing to the fact that the system architecture of both QBC and the quantum radar are identical, a functioning QBC system could be built by adapting recent realizations of the microwave sensing prototypes.

\section{CONCLUSIONS}

QBC constitutes a novel application of quantum radar technology that allows simple classical backscatter Tags to communicate with a quantumenhanced Reader/BS. Only the Reader/BS device has to operate at an ultra-low temperature, while the Tag can stay in the warm environment and use classical means to communicate. The potential exciting application of QBC includes secure transactions in banking applications (e.g., ATM using NFC to read a credit card) and ultra-low power RF communications in medical applications. QBC is still in its infancy and much research is needed to understand its performance limits and to develop both optimal theoretical protocols as well as protocols that can be 
implemented in practice. What is practical evolves all the time, since quantum memory and quantum computers are rapidly developing. A compelling potential application of QBC is to construct a direct wireless communication link between a quantum computer and its classical peripherals - do join this exciting research-journey, valued Colleague!

\section{ACKNOWLEDGMENT}

The work was supported in part by the Academy of Finland under Projects No. 311760 and No. 319578. L. Hanzo would like to acknowledge the financial support of the Engineering and Physical Sciences Research Council projects EP/Noo4558/1, EP/PO34284/1, COALESCE, of the Royal Society's Global Challenges Research Fund Grant as well as of the European Research Council's Advanced Fellow Grant QuantCom.

\section{REFERENCES}

[1] J. H. Shapiro, Z. Zhang, and F. N. C. Wong, "Secure communication via quantum illumination," Quantum Inf. Process., vol. 13, no. 10, pp. 2171-2193, Oct. 2014.

[2] C. Weedbrook, S. Pirandola, and T. C. Ralph, "Continuousvariable quantum key distribution using thermal states," Phys. Rev. A, vol. 86, p. 022318, Aug. 2012.

[3] P. Botsinis, Z. Babar, D. Alanis, D. Chandra, H. Nguyen, S. X. $\mathrm{Ng}$, and L. Hanzo, "Quantum error correction protects quantum search algorithms against decoherence," Scientific reports, vol. 6, p. 38095, 2016.

[4] R. Malaney, "The quantum car," IEEE Wireless Commun. Lett., vol. 5, no. 6, pp. 624-627, Dec. 2016.

[5] M. Malik, O. S. Magaña-Loaiza, and R. W. Boyd, "Quantumsecured imaging," Appl. Phys. Lett., vol. 101, no. 24, p. 241103, 2012.

[6] Z. Li, G. Xu, X. B. Chen, X. Sun, and Y. X. Yang, "Multi-user quantum wireless network communication based on multi-qubit GHZ state," IEEE Commun. Lett., vol. 20, no. 12, Dec. 2016.

[7] S. Lloyd, "Enhanced sensitivity of photodetection via quantum illumination," Science, vol. 321, no. 5895, pp. 1463-1465, 2008.

[8] S. Barzanjeh, S. Guha, C. Weedbrook, D. Vitali, J. H. Shapiro, and S. Pirandola, "Microwave quantum illumination," Phys. Rev. Lett., vol. 114, no. 8, p. 080503, 2015.

[9] M. Lanzagorta, "Quantum radar," Synthesis Lectures on Quantum Computing, vol. 3, no. 1, pp. 1-139, 2011.

[10] R. Jäntti, R. Di Candia, R. Duan, and K. Ruttik, "Multiantenna quantum backscatter communications," in Proc. IEEE Globecom Workshops, Singapore, Dec. 2017, pp. 1-6.

[11] R. Di Candia, R. Jäntti, R. Duan, J. Liétzen, H. Khalifa, and K. Ruttik, "Quantum backscatter communication: A new paradigm," in Proc. ISWCS, Lisbon, Portugal, Aug. 2018, pp. $1-6$.

[12] H. Khalifa and R. Jäntti, "Quantum backscatter communication with photon number states," in Proc. IEEE Globecom Workshops, Abu Dhabi, UAE, Dec. 2018.

[13] Q. Zhuang, Z. Zhang, and J. H. Shapiro, "Optimum mixedstate discrimination for noisy entanglement-enhanced sensing," Phys. Rev. Lett., vol. 118, p. 040801, Jan. 2017.
[14] M. Lanzagorta and J. Uhlmann, "Virtual modes for quantum illumination," in Proc. IEEE CAMA, Vasteras, Sweden, Sep. 2018, pp. 1-4.

[15] S. Barzanjeh, S. Pirandola, D. Vitali, and J. Fink, "Experimental microwave quantum illumination," arXiv preprint arXiv:1908.03058, 2019.

Riku Jäntti [SM'07] (riku.jantti@aalto.fi) received his D.Sc. degree (with distinction) in automation and systems technology in 2001. He is a full professor of communications engineering and the head of the Department of Communications and Networking at Aalto University. $\mathrm{He}$ is an associate editor of IEEE TVT. He is also IEEE VTS Distinguished Lecturer (Class 2016). His research interests include machine-type communications, cloud-based radio access networks, and quantum communications.

Ruifeng Duan [M'14] (ruifeng.duan@aalto.fi) received his M.Sc. and D.Sc.(tech.) degrees in 2008 and 2014, respectively. Since August 2014, he has been with Aalto University. His current research interests include extreme value theory, ambient backscatter communication, and ultra-reliable low latency communication.

Jari Lietzén (jari.lietzen@aalto.fi) received his M.Sc. degree (with distinction) in Communication Engineering from Aalto University in 2016. Currently he is pursuing doctoral studies at Aalto University. His research interests include error correction and key growing protocols in quantum key distribution and backscatter communications.

Hany Khalifa (hany.khalifa@aalto.fi) received his M.Sc. from Ain Shams University in 2018. Currently he is pursuing doctoral studies at Aalto University. His research interests include quantum optics, quantum information theory and quantum communications.

Lajos Hanzo [F'08] (lh@ecs.soton.ac.uk), FREng, FIET, Fellow of EURASIP, D.Sc., received his degree in electronics in 1976 and his doctorate in 1983. He holds honorary doctorates from the Technical University of Budapest (2009) and the University of Edinburgh (2015). He is a member of the Hungarian Academy of Sciences and a former Editor-in-Chief of IEEE Press. He is a Governor of both IEEE ComSoc and VTS. He has 30,000+ citations. 